\title{
How (not) to appeal
}

\author{
Authors can appeal editorial decisions, and editors will always consider each appeal carefully. However, not all \\ appeals are successful. Under what circumstances is appealing an editorial decision likely to reverse the outcome, \\ and what are the features of a strong appeal?
}

$\mathrm{R}$ ejections are always a disappointing outcome, and sometimes authors may feel that the rejection of their paper by the journal was unwarranted. At Nature Human Behaviour, we always consider the views of authors who feel that their work has been misjudged. Whether appeals lead to reversal of the original decision or not depends on the grounds for appeal and how these relate to the reason(s) the work was rejected. This is true regardless of whether the rejection was based purely on editorial considerations without peer review or was informed by reviewer feedback.

\section{When to appeal}

Enthusiasm for research drives science forward, and authors are understandably invested in their work and enthusiastic about their papers. So editorial rejections without the opportunity for peer feedback may feel particularly disappointing. For initial decisions, we read manuscripts in the context of their field and carefully consider the degree to which they fulfil the criteria that Nature Human Behaviour applies across all submissions (Nat. Hum. Behav. 3, 1127-1128; 2019). Appeals based on disagreements between editors and authors about novelty, significance, breadth of potential relevance or strength of evidence are very rarely successful.

In $2020,12 \%$ of appeals on editorial rejections were successful, and in the vast majority of cases the reversal of the original decision was motivated by the addition of data or analyses that strengthened support for the authors' conclusions or - less frequently - instances of genuine editorial error (for instance, where a particular prior publication we felt undermined the contribution a manuscript made to the literature turned out not to do so on further inspection).

There are a number of good reasons to appeal post-review and $32 \%$ of post-review appeals in 2020 were successful.

A rejection may be motivated by referee comments that suggest that a significant amount of additional data are needed to support the main claim of an article. If we feel the request for that additional data is well-founded but presents an unreasonable burden because the amount of additional work involved would require more than six months to complete, or constitutes more than $50 \%$ of the work already included, we will typically reject the paper. If, however, you already have or are willing to collect these data, that presents a strong ground for appeal.

Sometimes, editorial decision letters or reviewer reports contain factual errors. Perhaps specific aspects of the methods were misinterpreted, or their validity was misjudged. Where factual errors appear to have driven the decision, it is reasonable to appeal. Editors and reviewers make mistakes. Nonetheless, editors and reviewers are also experts in their line of work. Therefore, misunderstandings should motivate at least textual revisions, so that future readers are less likely to make similar mistakes.

Another reason that motivates potentially strong appeals is specific, concrete evidence of reviewer bias. These cases, however, are exceedingly rare. Reviewer reports that contain criticisms of the work that are not supported by evidence, or are directed at the field to which a manuscript belongs or at the authors (all signs of potential bias), do not factor into the editorial decision to reject a piece of work. Although we do not suppress reviewer reports that we perceive are based on unfounded or irrelevant criticisms of the work, our decisions are based on the input of reviewers whose evaluations are supported by evidence and well-thought-out arguments - and the decision letter will explain what these are. However, if, for example, a reviewer expressed deep concerns about the novelty of a finding or method and further investigation identified a significant reviewer conflict of interest in that regard, an appeal would be successful.

As for rejections without peer review, disagreements between authors and reviewers about the significance of the work and the contribution it makes to the literature are extremely unlikely to succeed.

\section{How to appeal}

Sleep on it. It is tempting to fire off an immediate response to a negative decision, but you will be doing yourself a disservice if you don't take the time to get past the initial emotional response a rejection causes. To maximize the chances of a successful appeal, talk to your co-authors and consider carefully the grounds, content and tone of your appeal before contacting your handling editor.

Present new data or analyses. 'Cosmetic' changes to your manuscript will not lead editors to overturn a decision - what we will be looking for are new data or analyses that address the substance of editors' or reviewers' concerns with your work. Do not change the nature of your hypotheses, but be transparent about what data do or do not inform these.

Argue scientifically. Rejections are frustrating, particularly those that feel unfair. A strong appeal is measured, engages constructively with the feedback and offers revisions to the manuscript to prevent future misjudgements and to address all valid points. Make scientific arguments; avoid ad hominem attacks.

Status is irrelevant. We view unfavourably appeals that rely on the authors' reputation or publication record as arguments for reconsideration of the work. We also do not take into consideration endorsements by notable colleagues shared with us by authors.

Don't ask to speak to the manager. We reach decisions as a team. All appeals are evaluated by more than one editor, as well as the chief editor of the journal. Asking for a different editor will not alter the decision on your manuscript.

Fundamentally, we as editors make decisions about research manuscripts, not people. A good appeal has the hallmarks of all good scientific writing: presenting strong evidence in a thoughtful way, engaging constructively with the criticisms and implementing meaningful revisions in response to editorial and/or referee feedback. For the same reasons, making arguments about people - yourself or others - is a weak strategy.

For rejections after review, we will typically ask for a revised manuscript and 
a point-by-point reply to the referees' comment before we decide whether we can reverse our previous decision.

In fairness to newly submitting authors, the evaluation of appeals necessarily has lower priority and all decisions on appeals are final (that is, we will not consider a second appeal of the same decision). Ultimately, most appeals are unsuccessful and require significant investment of time and effort on your behalf. If you wish to appeal a decision, consider carefully the strength of your case before making this investment to avoid unnecessary delays in the ultimate publication of your work.

Published online: 21 July 2021

https://doi.org/10.1038/s41562-021-01174-w 